Rev. Elev. Méd. vét. Pays trop., 1965, 18, 3 (307-312).

\title{
Pâturages artificiels en savanes à saison sèche peu marquée *
}

\author{
R. CADOT, J. COULOMB, R. RIVIËRE
}

\begin{abstract}
RÉSUMÉ
Après un bref résumé des conditions climatiques de la région de Bouaké (République de Côle-d'lyoire), less auteurs exposent les résultais obtenus par une étude comparée de quelques plantes introduites : techniques d'implantation, rythme d'exploitation, longévité des pâturages, productıvité el valeur fourragère.

Les auleurs indiquent ensuile quelques associations « graminées-légumineuses » susceptibles d'être utilisées pour les sols locaux.
\end{abstract}

Ces travaux ont été réalisés au centre de recherches zootechniques de Minankro près de Bouaké ( $7045^{\prime}$ de latitude Nord), en République de Côte-d'Ivoire.

Le climat de type Baoulé-Dahoméen d'Aubreville est caractérisé par une température relativement uniforme avec une moyenne annuelle de $26005 \mathrm{C}$ et une pluviosité de $1.207 \mathrm{~mm}$ en 95 jours.

L'indice des saisons pluviométriques d'Aubreville est 6.4 .2 (6 mois de plus de $100 \mathrm{~mm}$, deux mois recevant moins de $30 \mathrm{~mm}$ et 4 mols intermédıaires).

La grande saison sèche s'étale sur 4 mois, de novembre à février et une petıte saison sèche apparaît en juillet et août, permettant deuxcycles de cultures.

Le substratum géologique de granite alcalin a donné naissance à un plateau mollement ondulé et les sols de plateau sont des sols rouges ferrugineux tropicaux, acides et chimiquement pauvres, sauf en potasse dans les 20 premiers centimètres.

* Communication présentée au IX Congrès international des pâturages. SAO PAULO (Brésil). 7-20 Janvier 1965.
La végétation naturelle des plateaux est une savane arborée et arbustive à strates ligneuses denses.

\section{Expérimentation des pâturages artificiels.}

L'agriculture régionale est de type familial et son évolution actuelle est axée sur l'utilisation de bœufs en culture attelée. Les problèmes d'alimentation de ces boufs sont à l'origine des recherches entreprises sur les pâturages artificiels.

L'expérimentation comporte les phases suivantes:

10 Etablissement d'une collection :

393 espèces ou variétés locales ou introduites, ont été mises en observation pour étudier :

- Facilité d'implantation.

- Développement végétatif.

- Floraison.

- Fructification.

- Résistance à la sécheresse.

- Reprise de végétation au début des plules (pour les espèces pérennes).

- Résistance aux maladies et parasites.

$2^{\circ}$ Essais d'orientation des espèces les plus intéressantes afin d'étudier les rythmes d'exploitation et leur productivité. 
Quatre légumineuses et 16 graminées ont été étudiées :

\section{Graminées}

$\begin{array}{ll}\text { Andropogon gayanus } & \text { Melinis minutifiora } \\ \text { Cynodon plectostachyum } & \text { Brachiaria ruziziensis } \\ \text { Panicum maximum } & \text { Pennisetum merckere } \\ \text { Digitaria decumbens } & \text { Brachiaria mutica } \\ \text { Pennisetum purpureum. } & \text { Chloris gayana } \\ \text { Setaria sphacelata } & \text { Paspalum virgatum } \\ \text { Digitaria umfolozi } & \text { Pennisetum polystachyum } \\ \text { Dichanthium annulatum } & \text { Panicum antidotale }\end{array}$

\section{Légumineuses}

Desmodium asperum Desmodium tortuosum Centrosema pubescens Stylosanthes gracilis

\section{Dispositif :}

Chaque espèce figure sur trois parcelles de $7 \times 7 \mathrm{~m}$.

- une exploitée au stade pâture à une hauteur de végétation de 30 à $50 \mathrm{~cm}$ selon les espèces;

- une exploitée au stade fourrage àl'épiaison:

- une laissée en repos pour l'étude des fructifications.

Les prélèvements et pesées sont effectués sur des carrés intérieurs de $5 \times 5 \mathrm{~m}$ après élimınation d'une bordure de $1 \mathrm{~m}$.

Des échantillons sont soumis à l'analyse pour permettre les comparaisons entre les différentes espèces, les différents nıveaux d'exploitation

TABLEAU H० I a

Stade pature

\begin{tabular}{|c|c|c|c|c|}
\hline \multirow[b]{2}{*}{ Espèces } & \multirow{2}{*}{$\begin{array}{l}\text { Nombre } \\
\text { de } \\
\text { coupes }\end{array}$} & \multicolumn{3}{|c|}{ Rendement à I'hectare } \\
\hline & & $\begin{array}{l}\text { Poids en } \\
\text { tonnes }\end{array}$ & $\begin{array}{c}\text { Dnités } \\
\text { fourragères }\end{array}$ & $\begin{array}{c}\text { Matières } \\
\text { azotées } \\
\text { digestibles en kg }\end{array}$ \\
\hline Panicum maxamum & 10 & 87,7 & 14.040 & 1380 \\
\hline Andropogon gayanus & 10 & 71,5 & 16.420 & 1165 \\
\hline Stylosanthes ETacilia & 5 & 66,0 & 11.230 & 2115 \\
\hline Melunss minutiflore & 6 & 56.5 & 11.875 & 630 \\
\hline Centrosema pubeacens & 5 & 39,7 & 5.570 & 1310 \\
\hline Digiteria wnfolozi & 6 & 34,8 & 4.185 & 265 \\
\hline
\end{tabular}

TABLIAUU NO I b

Stade fourrage

\begin{tabular}{|c|c|c|c|c|}
\hline \multirow[b]{2}{*}{ Espèces } & \multirow{2}{*}{$\begin{array}{l}\text { Nonbre } \\
\text { de } \\
\text { coupes }\end{array}$} & \multicolumn{3}{|c|}{ Rendement à l'hectare } \\
\hline & & $\begin{array}{l}\text { Poids en } \\
\text { tonnes }\end{array}$ & $\begin{array}{c}\text { Unités } \\
\text { fourragères }\end{array}$ & $\begin{array}{c}\text { Matières } \\
\text { szotées } \\
\text { digestibles en } \mathrm{kg}\end{array}$ \\
\hline Paricum marimum & 7 & 90,5 & 19.920 & 1.060 \\
\hline Andropogon gayenus & 6 & 78,4 & 14.905 & 885 \\
\hline Stylosanthes gracills & 4 & 46,1 & 15.680 & 1.155 \\
\hline Melinis minutiflora & 4 & 56,0 & 11.760 & 480 \\
\hline Centroseme pubescens & 4 & 39,6 & 9.905 & 2.890 \\
\hline Digitaria umfolozi & 5 & 47,6 & 7.150 & 590 \\
\hline
\end{tabular}


pour une espèce, et suivre l'évolution de la plante selon la saison et lors du vieillissement du pâturage.

Les résultats d'analyse sont encore insuffisants pour permettre des conclusions quant à ces différents aspects de la production.

L'essai est mené sans apport d'engrais afin de suivre l'évolution naturelle du pâturage.

\section{Résultats.}

Sur une période d'une année, les résultats figurant dans les tableaux la et lb mettent en évidence :
- Le haut niveau de production du Panicum moximum et de l'Andropogon gayonus, tant sur le nombre d'exploitations que sur les tonnages et la valeur fourragère.

- Les productions intéressantes de Stylosanthes gracilis et Melinis minutiflora avec des rythmes d'exploitation semblables. Ces deux espèces pourraient donc être associées en culture.

- La meilleure productivité sous pâture d'Andropogon goyanus et Stylosanthes gracilis alors que Panicum maximum est meilleur comme fourrage.

Les tableaux llo et $\mathrm{ll} b$ mettent en relief la productıvité des espèces au cours de la saison

TABLELU NO II a

Stade pature

\begin{tabular}{|c|c|c|c|c|c|c|}
\hline \multirow[b]{2}{*}{ Espèces } & \multicolumn{4}{|c|}{$\begin{array}{l}\text { Saison sèche : } 141 \text { jours } \\
\text { (du 1.11.63 au } 20.3 .64 \text { ) }\end{array}$} & \multicolumn{2}{|c|}{$\begin{array}{l}\text { Saison des pluies } \\
224 \text { jours }\end{array}$} \\
\hline & $\begin{array}{l}\text { Nambre } \\
\text { de } \\
\text { coupes }\end{array}$ & $\begin{array}{l}\text { Poids en } \\
\text { tonines }\end{array}$ & $\begin{array}{l}\text { Unités } \\
\text { fourragères }\end{array}$ & $\begin{array}{l}\text { Matières } \\
\text { azotées } \\
\text { digestibles } \\
\text { en } \mathrm{kg}\end{array}$ & $\begin{array}{l}\text { Mombre } \\
\text { de } \\
\text { coupes }\end{array}$ & $\begin{array}{l}\text { Poida en } \\
\text { tonnes }\end{array}$ \\
\hline Andropogon gayanus & 3 & 20,6 & 3710 & 300 & 7 & 50,8 \\
\hline Paricum marimum & 2 & 15,0 & 1350 & 160 & 8 & 72.7 \\
\hline Stylosanthes gracilig & 1 & 7,2 & 1600 & 275 & 4 & 58,7 \\
\hline Melinis minutiflora & 1 & 12,8 & 2570 & 125 & 5 & 43.7 \\
\hline Centrosema pubescens & 1 & 10,4 & 1040 & 320 & 4 & 29,3 \\
\hline Digiteria umfolozi & 1 & 4,0 & 650 & 30 & 5 & 30,8 \\
\hline
\end{tabular}

TABLELU No II b

Stade fourrage

\begin{tabular}{|c|c|c|c|c|c|c|}
\hline \multirow[b]{2}{*}{ Espècea } & \multicolumn{4}{|c|}{$\begin{array}{l}\text { Saison sàche : } 141 \text { jours } \\
\text { (du 1.11.63 au 20.3.64) }\end{array}$} & \multicolumn{2}{|c|}{$\begin{array}{l}\text { Sejeon des pluites } \\
224 \text { jours }\end{array}$} \\
\hline & $\begin{array}{l}\text { Nombro } \\
\text { de } \\
\text { coupes }\end{array}$ & $\begin{array}{l}\text { Poldo en } \\
\text { tonnes }\end{array}$ & $\begin{array}{c}\text { Unités } \\
\text { fourragèrea }\end{array}$ & $\begin{array}{l}\text { Matières } \\
\text { azotées } \\
\text { digestibles } \\
\text { en kg }\end{array}$ & $\begin{array}{l}\text { Nombre } \\
\text { de } \\
\text { coupes }\end{array}$ & $\begin{array}{l}\text { Poids en } \\
\text { tonnes }\end{array}$ \\
\hline Andropogon geyanus & 3 & 23,9 & 4550 & 270 & 3 & 54,4 \\
\hline Paricum mardmum & 2 & 16,2 & 3910 & 140 & 5 & 74,2 \\
\hline Stylosanthes gracilis & 2 & 22,4 & 2240 & 345 & 2 & 23,6 \\
\hline Melinis minutiflora & 1 & 6,1 & 1665 & 60 & 3 & 49,8 \\
\hline Centrosems pubescens & 1 & 13 & 3250 & 950 & 3 & 26.6 \\
\hline Dightarie umfolozi & 2 & 14,2 & 2280 & 160 & 3 & 23.4 \\
\hline
\end{tabular}


TABIAHAU No III a

Stade pâture

\begin{tabular}{|c|c|c|c|c|c|c|c|c|}
\hline \multirow[b]{3}{*}{ Espèces } & \multirow{3}{*}{$\begin{array}{l}\text { Délai } \\
\text { mise en } \\
\text { place à } \\
\text { lère coupe } \\
\text { (jours) }\end{array}$} & \multirow{3}{*}{$\begin{array}{l}\text { Périodicité } \\
\text { moyenne } \\
\text { des coupes } \\
\text { (jours) }\end{array}$} & \multicolumn{4}{|c|}{ Productıvité } & \multicolumn{2}{|c|}{ Valeur fourragère } \\
\hline & & & \multicolumn{2}{|c|}{ †ère année } & \multicolumn{2}{|c|}{ 2èrne année } & \multirow{2}{*}{$\begin{array}{c}\text { Unités } \\
\text { fourragères } \\
\text { par kg }\end{array}$} & \multirow{2}{*}{$\begin{array}{l}\text { Matières } \\
\text { azotées } \\
\text { digestioles } \\
\text { on } \mathrm{g} / \mathrm{kg}\end{array}$} \\
\hline & & & $\begin{array}{l}\text { Folds } \\
\text { (tonnes) }\end{array}$ & $\begin{array}{l}\text { Nombre } \\
\text { de } \\
\text { colpes }\end{array}$ & $\begin{array}{l}\text { Poids } \\
\text { (tonnes) }\end{array}$ & $\begin{array}{l}\text { Nombre } \\
\text { de } \\
\text { coupes }\end{array}$ & & \\
\hline Panicum me iraun & 61 & 35 & 82,8 & 11 & 91,8 & 10 & 0,11 & 21,5 \\
\hline Digitaria unfolozi & 52 & 61 & 65,7 & 7 & 34,8 & 6 & 0,12 & 7,6 \\
\hline Andropogon gayanus & 74 & 50 & 39,5 & 7 & 42,2 & 8 & 0,14 & 14.2 \\
\hline Centrosema pubescens & 110 & 104 & 20,2 & 2 & 39.7 & 5 & 0,14 & 32,6 \\
\hline Melinis muntiflora & 66 & 72 & 23 & 5 & 26,5 & 5 & 0,20 & 8,1 \\
\hline
\end{tabular}

TABLFAUU NO III D

Stade fourrage

\begin{tabular}{|c|c|c|c|c|c|c|c|c|}
\hline \multirow[b]{3}{*}{ Espèces } & \multirow{3}{*}{$\begin{array}{c}\text { Délai } \\
\text { mise en } \\
\text { place à } \\
\text { lère coupe } \\
\text { (jours) }\end{array}$} & \multirow{3}{*}{$\begin{array}{l}\text { Péruodicité } \\
\text { moyenne } \\
\text { des coupes } \\
\text { (jours) }\end{array}$} & \multicolumn{4}{|c|}{ Productivité } & \multicolumn{2}{|c|}{ Valeur fourragkere } \\
\hline & & & \multicolumn{2}{|c|}{ tère année } & \multicolumn{2}{|c|}{ 2ème année } & \multirow{2}{*}{$\begin{array}{l}\text { Unités } \\
\text { fourragères } \\
\text { par ke }\end{array}$} & \multirow{2}{*}{$\begin{array}{l}\text { Matières } \\
\text { azotées } \\
\text { digestibles } \\
\text { en } \mathrm{g} / \mathrm{kg}\end{array}$} \\
\hline & & & $\begin{array}{l}\text { Poids } \\
\text { (tonnes) }\end{array}$ & $\begin{array}{c}\text { Nombre } \\
\text { de } \\
\text { coupes }\end{array}$ & $\begin{array}{c}\text { Poids } \\
\text { (tomnes) }\end{array}$ & $\begin{array}{l}\text { Nombre } \\
\text { de } \\
\text { coupes }\end{array}$ & & \\
\hline Panicum maxinaum & 75 & 45 & 96,7 & 10 & 81,7 & 7 & 0,19 & 15,6 \\
\hline Digitaria umfolozi & 41 & 75 & 70,2 & 4 & 47,6 & 5 & 0,15 & 12,4 \\
\hline Andropogon gajanus & 84 & 63 & 42,5 & 7 & 60,3 & 5 & 0,17 & 8,8 \\
\hline Centrosema pubescens & 110 & 104 & 30,4 & 2 & 26,0 & 3 & 0,25 & 73 \\
\hline Melinis minutiflors & 66 & 102 & 50,0 & 4 & 44,7 & 3 & 0,17 & 6.5 \\
\hline
\end{tabular}


sèche, l'exploitation étant poursuivie toute l'année.

Andropogon goyonus ef Ponicum maximum sont encore les plus intéressants avec un temps de repos entre deux pacages d'un mois et demi à deux mois en saison sèche et un mois en saison des pluies.

Le temps de repos entre deux coupes pour fourrage est plus élevé en saison des pluies ( 1 mois et demi).

Les tableaux Illa ef Illb précisent la productivité obtenve sur deux années consécutives et Panicum maximum confirme sa haute production avec possibilité de l'exploiter très vite après son implantation par éclats de souche.

$3^{\circ}$ Essais de charge destinés à confirmer par pacage les résultats obtenus en essais d'orientation.

Ces essais sont en cours sur des parcelles d'un quart d'hectare avec les espèces ou mélanges suivants :
Ponicum maximum

Andropogon goyonus

Digitorio umfolozi

Melinis minutiflora

Dichonthium annulatum

Cynodon plectosfachyum

Ces essais ont pour but de préciser :

- le rythme d'exploitation en pâturage,

- les modalités d'exploitatıon.
Stylosanthes gracilis Melinis minutifiora $\times$ Stylosanthes Centrosemo pubescens Andropogon gayanus $\times$ Stylosanthes

Setaria sphacelato

\section{Conclusion.}

D'après les premiers résultats obtenus, les possibilités d'affouragement en pâturages artificiels semblent très favorables dans la moyenne Côte-d'Ivoire avec un nombre d'espèces fourragères suffisant.

Les associations de graminées et légumineuses posent des problèmes d'équilibre non résolus, problèmes dus au rythme d'exploitation propre à chaque espèce et à leur appétibilité relative.

Mais I'association Melinis minutifloro et Stylosanthes gracilis semble possible, leur rythme de productivité étant très voisin.

Avec les résultats obtenus en essais d'orientation, on peut envisager en pâturage rationné, l'entretien de deux paires de bceufs à l'ha sur Panicum maximum et une paire de bœufs sur Digitoria umfolozı, mais l'expérimentation avec pacage périodique est indispensable pour vérifier ces hypothèses.

\section{SUMMARY}

\section{Artificial pasture-lands in stightly dry season savannahs}

\footnotetext{
After a brief review on the climalic conditions of the Bouake country (IvoryCoast republic), the authors report their results obtained in the comparative study of some introduced plants : implantation technics, exploitation rhythms, meadows longevity, produchivity and fodder value.

The authors indicate some associations graminaceous-leguminous plants which ore abble to be joined in the local rotations.
} 


\section{RESUMEN}

Paslos artificiales en sábanas de estación seca poco importante

Después de un breve resúmen de las condiciones climáticas de la region de Bouake (Rep. de la Costa de Marfil), los autores exponen los resultados obtenidos en un esfudio comparativo de algunas plantas introducidas : técnicas de implantación, ritmo de explotación, longevidad de los pastos, productividad y valor forrajero.

Los autores indican a continuación algunas asociaciones «gramineasleguminosas » susceptibles de ser integradas en los suelos locales. 